\title{
Auxerre. Suivi archéologique du chantier de restauration des toitures de la nef de la cathédrale Saint-Étienne
}

Sylvain Aumard

\section{(2) OpenEdition}

Journals

Édition électronique

URL : https://journals.openedition.org/cem/2802

DOI : $10.4000 /$ cem.2802

ISSN : 1954-3093

Éditeur

Centre d'études médiévales Saint-Germain d'Auxerre

Édition imprimée

Date de publication : 15 août 2003

ISSN : 1623-5770

Référence électronique

Sylvain Aumard, «Auxerre. Suivi archéologique du chantier de restauration des toitures de la nef de la cathédrale Saint-Étienne », Bulletin du centre d'études médiévales d'Auxerre / BUCEMA [En ligne], 7 | 2003, mis en ligne le 16 juin 2009, consulté le 22 septembre 2022. URL : http://journals.openedition.org/ cem/2802 ; DOI : https://doi.org/10.4000/cem.2802

Ce document a été généré automatiquement le 22 septembre 2022.

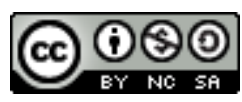

Creative Commons - Attribution - Pas d'Utilisation Commerciale - Partage dans les Mêmes Conditions 4.0 International - CC BY-NC-SA 4.0

https://creativecommons.org/licenses/by-nc-sa/4.0/ 


\title{
Auxerre. Suivi archéologique du chantier de restauration des toitures de la nef de la cathédrale Saint-Étienne
}

\author{
Sylvain Aumard
}

\section{Le chantier}

1 Le chantier de restauration de la cathédrale a commencé en avril 2002 par la toiture de la nef. La mise en place du Conseil scientifique allait permettre de s'intéresser de très près à un terrain un peu nouveau pour le Centre, plutôt habitué à intervenir sur des maçonneries que sur des charpentes et des couvertures. Le déroulement et la teneur du suivi archéologique ont été adaptés à la progression du chantier de restauration, en trois tranches, d'est en ouest. Les observations ont porté à chaque fois sur la dépose des tuiles anciennes (jetées ou stockées pour la Ville selon leur état) et sur le démontage des pièces de charpentes à remplacer (sauf la première tranche).

2 Ces investigations se sont déroulées sans perturber le travail des différentes entreprises. En outre, celles-ci se sont largement prêtées au jeu de notre collaboration grâce à la qualité du dialogue qui s'est engagé au fil des travaux. Ces contacts permanents ont parfois été les garants de certains résultats, notamment en permettant de s'accorder sur le planning ou d'échanger certains points de vue et observations diverses.

3 En plus de nos propres travaux, deux étudiants (Laurent Durnecker et Alexandra Weber) de l'Université de Bourgogne, placés sous la responsabilité de Denis Cailleaux, ont effectué des recherches en archives et ont procédé à des comptages au sein des stocks de la Ville. Certains aspects de ces recherches sont en cours de publication ${ }^{1}$ et feront l'objet d'un article de synthèse dans leur ensemble. 


\section{La dépose des tuiles}

4 Dans l'état de la couverture au moment de sa dépose, aucune tuile ne paraissait former un quelconque motif, malgré la présence - facilement repérable - d'éléments vernissés de couleur. La majeure partie de l'édifice présentait un aspect moucheté résultant des différentes campagnes de réfection et de repiquage. Ces premiers constats nous ont conduit à nous consacrer uniquement à la mise en place d'une typologie des tuiles, tâche difficile pour laquelle nous ne pouvions guère compter sur d'autres expériences comparables à une telle échelle.

5 Ce travail a permis de distinguer une douzaine de types de tuile d'après leur mode d'accrochage (absence/présence de crochet) et de fixation (trous pour des clous), leur forme et leurs dimensions. Deux catégories se distinguent particulièrement par la grande homogénéité de leurs échantillons qui tranche beaucoup avec les autres, aux modules assez diversifiés : les tuiles à chevilles (sans crochet) et les tuiles à crochets vernissées. Si cette diversité semble illustrer les différentes campagnes de réparation et de repiquage de la toiture, les effets de standardisation pourraient, à l'inverse, correspondre vraisemblablement à des commandes effectuées pour les chantiers médiévaux, d'autant qu'il s'agit d'objets connus pour la période qui nous intéresse.

Des datations en laboratoire (archéomagnétisme ${ }^{2}$ sur les tuiles et thermoluminescence ${ }^{3}$ sur la douzaine de faîtières à boutons glaçurées) devraient permettre de bien cerner la cohérence chronologique des éléments étudiés avec les autres données l'édifice.

\section{La charpente}

7 Les sablières et les blochets ont été refaits systématiquement à neuf car ces pièces ont subi l'action conjuguée de l'humidité et du poids de l'ouvrage. Au départ, notre inexpérience dans ce domaine nous avait conduit à négliger la surveillance de la première tranche correspondant à peu près au premier chantier daté de $1356{ }^{4}$. C'est à force de persévérance et, surtout, grâce à la décisive et fructueuse collaboration de Stefan King, de l'équipe allemande de Bauforschunger conduite par Heike Hansen en septembre 2002, que le regard porté sur l'édifice a pu évoluer et, par là, le choix des interventions archéologiques. Tout d'abord une couverture photographique a permis de documenter les nouvelles observations :

- la fin du chantier de la charpente du transept nord portant traces de machineries ;

- les traces de la croisée primitive intégrées dans l'ouvrage actuel ;

- les témoins de plusieurs cloisons provisoires (bardeaux sur pans de bois) à la jonction des différents chantiers successifs.

Une nouvelle étude dendrochronologique a été préparée, elle est programmée fin janvier 2003. Pour ce faire, nous avons repéré - en coordination avec les charpentiers les futurs éléments déposés et les avons stockés au fur et à mesure des restaurations. Le travail de ces derniers visait à compléter les premières analyses ${ }^{5}$, à savoir :

- dater les deux phases de la croisée de transept (carottages);

- compléter le corpus des données sur la nef par l'échantillonnage systématique (tronçonnages) des pièces de bois déposées. Il s'agissait ici, outre d'affiner les datations, de mener une action conservatoire sur des informations contenues dans les pièce de bois et qui allaient disparaître (informations sur le climat, sur l'aubier médiéval...) ; 
- suite aux observations faites au cours des travaux de relevé de la façade, dater le linteau en bois supportant la cloison provisoire installée à l'emplacement du tympan du portail nord.

Parallèlement au déroulement de la phase de restauration de la charpente (janviermars 2003), des relevés ont été entrepris par nos soins dans deux contextes aux problématiques différentes :

- d'une part le laisonnement entre les deux chantiers médiévaux du XIV siècle, qui devait disparaître au cours de la restauration, a été relevé au $1 / 10^{\mathrm{e}}$ (plan et coupe) afin d'en conserver une trace documentaire ; à notre demande, un élément de renfort a été conservé ${ }^{6}$;

- d'autre part, des secteurs inaccessibles situés au niveau du faîtage ont révélé des aspects particuliers non menacés mais observables dans les conditions exceptionnelles qu'offre un tel chantier ; c'est ce qui a motivé les relevés au $1 / 5^{\mathrm{e}}$ des bardeaux témoignant de la cloison provisoire installée à la fin du chantier de 1378 et le relevé in situ d'une grosse cheville en fer médiévale (dans la phase de 1378).

Cette première tranche de restauration se termine. Nous espérons vivement pouvoir reconduire cette approche à l'occasion des prochaines tranches de restauration. À l'heure actuelle, les reins de voûtes de la nef font l'objet d'un déblaiement complet, suivi par nos soins, motivé par la forte probabilité d'y trouver des indices sur les premières couvertures. Nous espérons là aussi donner la preuve de tout l'intérêt de notre démarche.

11 Sans attendre les résultats des laboratoires, on peut déjà affirmer que ces investigations, en étroite collaboration avec le maître d'ouvrage, les architectes et les entreprises, ont apporté un lot considérable d'informations sur l'histoire de la toiture de la cathédrale Saint-Étienne. C'est aussi et une nouvelle fois l'occasion de démontrer l'intérêt - et même la nécessité - de cette démarche archéologique ; une démarche qui ne vaut plus seulement pour le sous-sol ou les élévations et qui n'a pas seulement une valeur préventive ou conservatoire (pour garder en mémoire des informations en cours d'érosion), mais aussi une valeur scientifique à part entière en permettant de révéler au grand jour des aspects méconnus et inédits grâce à des conditions d'accessibilité et d'observations rares, voire exceptionnelles.

\section{NOTES}

1. S. AUMARD, D. CAIlleauX, L. DURneCKer, A. LeRiche et A. WEBER, "Tuiles vernissées et toitures ornées des édifices gothiques du nord de la Bourgogne. Premiers résultats des enquêtes sur les couvertures anciennes des cathédrales de Sens et d'Auxerre", Projet collectif de recherche "Terres cuites architecturales médiévales et modernes en île-de-France et dans les régions voisines (Centre, Normandie, Picardie, Champagne-Ardennes, Bourgogne)", sous la dir. de J. CHAPELOT (à paraître).

2. Laboratoire d'archéomagnétisme de l'Université de Rennes/CNRS, Philippe LANOS.

3. Institut supérieur industriel de Bruxelles (ISIB), Joseph GUILLAUME.

4. C. LocAtelli, C. LAVIER, "Les charpentes de l'ancien palais synodal et de la cathédrale SaintÉtienne à Auxerre", P. HOFFSUMMER (dir.), Les charpentes du XIe au XIXe siècle. Typologie et évolution 
en France du Nord et en Belgique, Paris, Monum./Éd. du Patrimoine (Cahiers du Patrimoine, $\mathrm{n}^{\circ}$ 62), 2002, p. 142-145. D'est en ouest, ont été mis en évidence un premier module de 27 fermes daté de 1356, puis un second de 9 fermes daté de 1378 et un troisième de 23 fermes daté de 1512.

5. La première série d'analyses avait surtout pour objectif de dater les principaux chantiers dans la finalité typologique de l'ouvrage paru récemment aux Éditions du Patrimoine (Les charpentes..., op. cit.). Cette nouvelle opération a été réalisée par Didier Pousset et Christine Locatelli (Centre régional de restauration et de conservation des œuvres d'art de Vesoul), doctorants au Laboratoire de chrono-écologie de Besançon (UMR 6565), avec la participation de Vincent Driget. 6. À notre regret, l'élément de renfort a seulement été préservé à côté de son emplacement primitif (donc susceptible de disparaître un jour) et non replacé dans sa position d'origine (coincé entre les arbalétriers et les jambettes).

\section{INDEX}

Index géographique : France/Auxerre

Mots-clés : cathédrale, Saint-Etienne d'Auxerre, toiture 